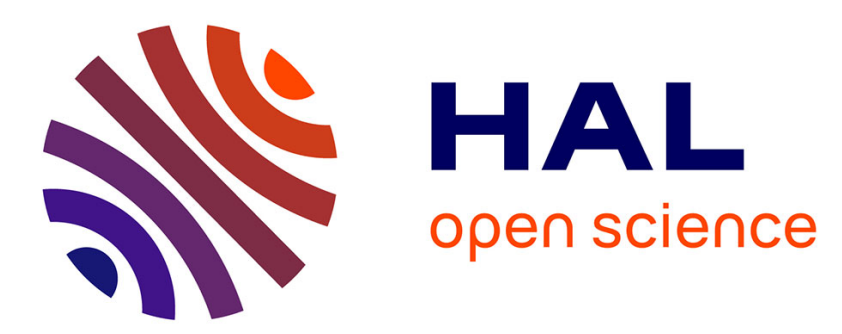

\title{
Effect of Waveform on Tactile Perception by Electrovibration Displayed on Touch Screens
}

Yasemin Vardar, Burak Güçlü, Cagatay Basdogan

\section{To cite this version:}

Yasemin Vardar, Burak Güçlü, Cagatay Basdogan. Effect of Waveform on Tactile Perception by Electrovibration Displayed on Touch Screens. IEEE Transactions on Haptics (ToH), 2017, 10 (4), pp.488-499. 10.1109/TOH.2017.2704603 . hal-03176863

\section{HAL Id: hal-03176863 https://hal.science/hal-03176863}

Submitted on 22 Mar 2021

HAL is a multi-disciplinary open access archive for the deposit and dissemination of scientific research documents, whether they are published or not. The documents may come from teaching and research institutions in France or abroad, or from public or private research centers.
L'archive ouverte pluridisciplinaire HAL, est destinée au dépôt et à la diffusion de documents scientifiques de niveau recherche, publiés ou non, émanant des établissements d'enseignement et de recherche français ou étrangers, des laboratoires publics ou privés. 


\title{
Effect of Waveform on Tactile Perception by Electrovibration Displayed on Touch Screens
}

\author{
Yasemin Vardar, Student Member, IEEE, Burak Güçlü, and Cagatay Basdogan, Senior Member, IEEE
}

\begin{abstract}
In this study, we investigated the effect of input voltage waveform on our haptic perception of electrovibration on touch screens. Through psychophysical experiments performed with eight subjects, we first measured the detection thresholds of electrovibration stimuli generated by sinusoidal and square voltages at various fundamental frequencies. We observed that the subjects were more sensitive to stimuli generated by square wave voltage than sinusoidal one for frequencies lower than $60 \mathrm{~Hz}$. Using Matlab simulations, we showed that the sensation difference of waveforms in low fundamental frequencies occurred due to the frequency-dependent electrical properties of human skin and human tactile sensitivity. To validate our simulations, we conducted a second experiment with another group of eight subjects. We first actuated the touch screen at the threshold voltages estimated in the first experiment and then measured the contact force and acceleration acting on the index fingers of the subjects moving on the screen with a constant speed. We analyzed the collected data in the frequency domain using the human vibrotactile sensitivity curve. The results suggested that Pacinian channel was the primary psychophysical channel in the detection of the electrovibration stimuli caused by all the square-wave inputs tested in this study. We also observed that the measured force and acceleration data were affected by finger speed in a complex manner suggesting that it may also affect our haptic perception accordingly.
\end{abstract}

Index Terms-Electrovibration, waveform, detection, tactile perception, psychophysical experiments, force, acceleration, touch screen

\section{INTRODUCTION}

C APACITIVE touch screens are indispensable part of smart phones, tablets, kiosks, and laptop computers nowadays. They are used to detect our finger position and enable us to interact with text, images, and data displayed by the above devices. To further improve these interactions, there is a growing interest in research community for displaying active tactile feedback to users through the capacitive screens. One approach followed for this purpose is to control the friction force between fingerpad of user and the screen via electrostatic actuation [1], [2], [3]. If an alternating voltage is applied to the conductive layer of a touch screen, an attraction force is generated between the finger and its surface. This force modulates the friction between the surface and the skin of the finger moving on it. Hence, one can generate different haptic effects on a touch screen by controlling the amplitude, frequency and waveform of this input voltage [1], [4], [5].

The electrical attraction between human skin and a charged surface was first reported by Johnsen and Rahbek in 1923 [6]. Around thirty years later, Mallinckrodt discovered that applying alternating voltages to an insulated aluminum plate can increase friction during touch and create a strange resin-like feeling [7]. He explained this phenomenon based on the well-known principle of parallel-plate capacitor. Later, Grimnes named this phenomenon as "electrovibration" and reported that surface roughness and dryness of finger skin could affect the perceived haptic effects [8]. Af-

- Manuscript received February 21, 2017; accepted May 9, 2017.

- Y. Vardar and C. Basdogan are with the Department of Mechanical Engineering, Koç University, Istanbul, Turkey, 34450.

E-mail: yvardar13@ku.edu.tr, cbasdogan@ku.edu.tr

- B. Güçlü is with Institute of Biomedical Engineering, Boğaziçi University, Istanbul, Turkey, 34684.

Email: burak.guclu@boun.edu.tr terwards, Strong and Troxel [9] developed an electrotactile display consisting of an array of electrodes insulated with a thin layer of dielectric. Using friction induced by electrostatic attraction force, they generated texture sensations on the touch surface. Their experimental results showed that the intensity of touch sensation was primarily due to the applied voltage rather than the current density. Beebe et al. [10], developed a polyimide-on-silicon electrostatic fingertip tactile display using lithographic microfabrication. They were able to generate tactile sensations on this thin and durable display using 200-600 V voltage pulses and reported the perception at the fingertip as "sticky." Later, Tang and Beebe [11] performed experiments of detection threshold, line separation and pattern recognition with visually impaired subjects. Although they encountered problems such as dielectric breakdown and sensor degradation, the subjects were able to differentiate simple tactile patterns by haptic exploration. Agarwal et. al [12] investigated the effect of dielectric thickness on haptic perception during electrostatic stimulation. Their results showed that variations in dielectric thickness had little effect on the threshold voltage. Kaczmarek et al. [13] explored the perceptual sensitivity of the human finger to positive and negative input pulses. Their results showed that the subjects perceived negative or biphasic pulses better than positive ones. In all of the above studies, electrovibration was obtained using opaque patterns of electrodes on small scale surfaces. However, in the recent works of Bau et al. [1] and Linjama et al. [2], electrovibration was delivered via a transparent electrode on a large commercial touch surface, which demonstrates the viability of this technology on mobile applications. Bau et al. measured the sensory thresholds of electrovibration using sinusoidal inputs applied at different frequencies [1]. They showed that the change in threshold voltage as a function 
of frequency followed a U-shaped curve similar to the one observed in vibrotactile studies. Later, Wijekoon et al. [4], followed the work of [2], and investigated the perceived intensity of modulated friction generated by electrovibration. Their experimental results showed that the perceived intensity was logarithmically proportional to the amplitude of the applied voltage signal.

To understand how mechanical forces develop at fingertip-surface interface, Mayer et al. [14], developed a tribometer and measured the lateral force to estimate the electrostatic attraction force for the applied voltage. They showed the effect of actuation frequency on the lateral frictional force despite some subject-dependent variability. They reported that this person to person variability highly depends on varying environmental impedances caused by voltage controlled electrovibration. Later, Vezzoli et al. [15] improved the model of electrovibration by including frequency-dependent electrical properties of human skin as documented in [16]. Recently, Kim et al. [17], suggested a method based on current control to solve the nonuniform intensity problem and developed a hardware prototype working with this principle. The results of their user study showed that the proposed current control method can provide more uniform intensity of electrovibration than voltage controlled one.

Although electrovibration can potentially provide rich tactile sensations, the number of applications of this technology is limited yet due to our poor understanding of the electrical and mechanical properties of human finger and its interaction with a touch surface. For example, both the electrical and mechanical impedances of the human finger are frequency-dependent, and the coupling between them has not been well understood yet [1], [14], [15], [17]. Moreover, human to human variability of these properties and the influence of the environmental factors on these properties further complicate the problem.

In addition to the physical factors mentioned above, it is known that human tactile (mechanical) perception varies with stimulation amplitude and frequency [18]. Even though the effects of amplitude and frequency on the human tactile perception of electrovibration have already been investigated using pure sine waves [1], there is no earlier study on how our perception changes when another waveform is used. In this paper, we investigate how input voltage waveform alters human haptic perception of electrovibration. This work is mainly motivated by our initial observation that square-wave excitation causes stronger vibratory sensation than sine-wave excitation. According to the parallel-plate capacitor principle, the electrostatic force is proportional to the square of the input voltage signal, hence the electrostatic force generated by a square-wave is supposed to be constant [19], [5]. Since DC (constant) excitation voltages do not cause vibration sensation (though it causes adhesion sensation as reported in [6], [20]), the square wave excitation is expected to be filtered electrically by the stratum corneum. This filtering suppresses the lowfrequency components in the excitation voltage and generates an electrostatic force with a distorted waveform. We hypothesise that the stronger vibratory sensation caused by a square wave is due to the high-frequency components in the resulting force signal. Since this waveform is rather complex (contains many frequency components), it can activate different psychophysical channels at different threshold levels [18], [21]. These four psychophysical channels (NPI, NPII, NPIII, P) are mediated by four corresponding mechanoreceptors and enable tactile perception. To predict tactile sensitivity, the Fourier components of the waveform should be analyzed by considering human sensitivity curve [18].

In this paper, using a simulation model developed in Matlab-Simulink, we first show that the forces displayed to human finger by electrovibration are very different for square and sinusoidal input voltages at low fundamental frequencies due to electrical filtering. Then, we show that the force waveform generated by square-wave excitation contains high-frequency components to which human tactile sensation is more sensitive. We support this claim by presenting the results of two experiments conducted with eight subjects. In the first experiment, we measure the detection threshold voltages for sinusoidal and square signals at various frequencies. In the second experiment, we actuate the touch screen at those threshold voltages and measure the contact force and acceleration acting on the index finger of subjects moving on the touch screen with a constant speed. We analyze the collected data in frequency domain by taking into account the human sensitivity curve and show that the square wave excites mainly Pacinian channel [22], [23]. Our results also suggest that scan speed has a significant effect on measured acceleration and force data and potentially on our haptic perception.

\section{WaVeform Analysis of Electrovibration}

Based on the well-known principle of parallel-plate capacitance effect [19], the electrostatic force acting on a human fingertip placed on a touch screen can be estimated as

$$
F_{e}=\frac{\epsilon_{0} \epsilon_{s c} A}{2}\left(\frac{V_{s c}}{d_{s c}}\right)^{2},
$$

where $\epsilon_{s c}$ is the relative permittivity of the stratum corneum, $\epsilon_{0}$ is the permittivity of vacuum, $A$ is the area of the fingerpad, $d_{s c}$ is the thickness of the stratum corneum. $V_{s c}$ is the voltage across the stratum corneum, which can be expressed as a function of the voltage applied to the conductive layer of the touch screen, $V$, as

$$
V_{s c}=V \frac{Z_{s c}}{Z_{b o d y}+Z_{s c}+Z_{a i r}+Z_{i}},
$$

where, $Z_{b o d y}, Z_{s c}, Z_{a i r}$, and $Z_{i}$ represent the impedances of the human body, stratum corneum, air gap and touch surface respectively (see Fig. 1).

The electrostatic force formulas given in [14], [15] are slightly different than Equation 1. In those articles, the authors expressed the perceived electrostatic force as the force generated between the conductive layer of the touch screen and human finger. More recently, Shultz et al. [20] derived this formula according to the voltage difference across the air gap (i.e gap between human fingertip and the insulator layer of the touch screen). However, in our opinion, the perceived tactile effects due to the electrostatic forces occur at the inner boundary of the stratum corneum, 


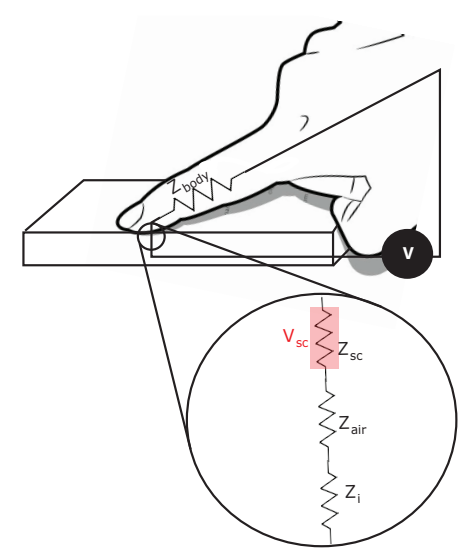

Fig. 1. An electrical model of human finger on a touch screen.

as the mechanoreceptors are located close to the epidermal junction or in the dermis [24], [25], [26]. Hence, we used $V_{s c}$ and not $V$ in our calculations. The reader may refer to [19] for more information related to the derivation of the electrostatic force generated at the boundaries of two parallel or series dielectrics.

To investigate the effect of waveform in electrovibration, we developed an equivalent circuit model of human finger in Matlab-Simulink environment [5]. In this model, we neglected the capacitance of the human body and air gap and also the internal resistance of the touch screen. The capacitance of the touch screen was calculated based on the properties of a commercial touch screen (3M Inc.), which was also used in our experiments ${ }^{1}$. Previous studies showed that the human skin (especially sweat ducts and the stratum corneum) is not a perfect dielectric and has frequencydependent resistive properties [13], [16], [27], [28]. Therefore, we modelled stratum corneum as a resistance and a capacitance in parallel. In [15], Vezzoli et al. used frequencydependent values of resistivity, $\rho_{s c}$, and dielectric constant, $\epsilon_{s c}$, of human stratum corneum reported by [16]. Their simulations showed that intensity of electrovibration was highly frequency-dependent. Similarly, we fitted polynomial functions to the experimental data reported by [16] and used those functions in our Matlab simulations (see Fig. 2a). Table 1 tabulates the parameters used in our model. For more information regarding this model, the reader may refer to [5].

Fig. $2 \mathrm{~b}$ represents the Bode plot of the transfer function $\frac{V_{s c}(s)}{V(s)}$, estimated by using the values tabulated in Table 1. The system displays the behavior of a bandpass filter with cut-off frequencies, $f_{\text {low }}$, and, $f_{\text {high }}$, at approximately $1 \mathrm{kHz}$ and $20 \mathrm{kHz}$ respectively. Hence, it shows a first order high pass filter behaviour up to $1 \mathrm{kHz}$, which can cause distortions on the voltage that is transmitted to stratum corneum at low frequencies.

To test the effects of this electrical filtering, we performed simulations with two different input waveforms (sinusoidal and square) at two fundamental frequencies (15 and 480

1. This touch screen is originally designed for capacitive-based touch sensing and composed of a transparent conductive sheet coated with an insulator layer on top of a glass plate. To generate haptic effects via electrovibration, the conductive sheet is excited by applying a voltage signal through the connectors designed for position sensing [1].
TABLE 1

The description of the parameters used in the circuit model and the corresponding values used in the Matlab simulations.

\begin{tabular}{|c||c||c||c|}
\hline Parameter & Explanation & Value & Unit \\
\hline \hline A & $\begin{array}{c}\text { Area of the } \\
\text { human fingertip }\end{array}$ & 1 & $\mathrm{~cm}^{2}$ \\
\hline$\epsilon_{0}$ & Permittivity of vacuum & $8.854 \times 10^{-12}$ & $F / m$ \\
\hline$R_{b o d y}$ & Resistance of human body [17] & 1 & $k \Omega$ \\
\hline$C_{i}$ & $\begin{array}{c}\text { Capacitance of the } \\
\text { 3M MicroTouch }\end{array}$ & $C_{i}=\frac{\epsilon_{0} \epsilon_{i} A}{d_{i}}$ & $F$ \\
\hline$\epsilon_{i}$ & $\begin{array}{c}\text { Relative permittivity of } \\
\text { the insulator }\end{array}$ & 3.9 & - \\
\hline$d_{i}$ & Thickness of the insulator & 1 & $\mu m$ \\
\hline$R_{s c}$ & Resistance of stratum corneum & $R_{s c}=\frac{\rho_{s c} d_{s c}}{A}$ & $\Omega$ \\
\hline$C_{s c}$ & Capacitance of stratum corneum & $C_{s c}=\frac{\epsilon_{0} \epsilon_{s c} A}{d_{s c}}$ & $F$ \\
\hline$\rho_{s c}$ & Resistivity of stratum corneum & Fig. 2a & $\Omega m$ \\
\hline$\epsilon_{s c}$ & $\begin{array}{c}\text { Relative permittivity of } \\
\text { the stratum corneum }\end{array}$ & Fig. 2a & - \\
\hline
\end{tabular}

$\mathrm{Hz}$ ). Fig. 3 shows the input voltage signal, the voltage across stratum corneum (filtered signals), and the resultant electrostatic force transmitted to mechanoreceptors for both waveforms at low and high frequencies (Figs. 3a and 3b). In low-frequency case $(15 \mathrm{~Hz})$, when the input is a sinusoidal signal, the output force signal is phase-shifted, and its amplitude drops significantly. Whereas, for a square wave signal, the output contains exponentially decaying relatively

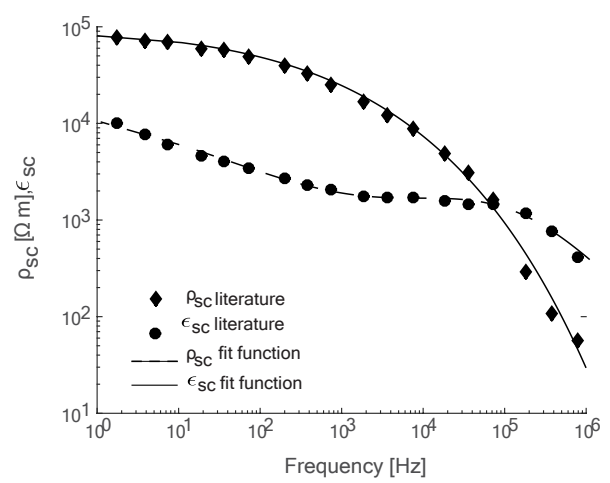

(a)
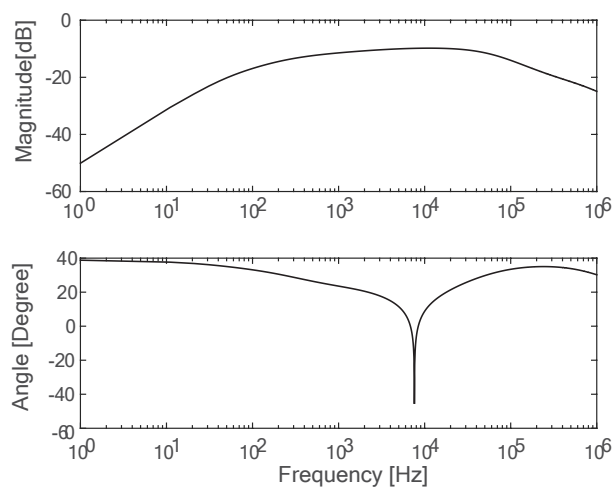

(b)

Fig. 2. a. The experimental values of resistivity and dielectric constant of stratum corneum as reported in [16] and the polynomial functions fitted to them. b. The transfer function between $V_{s c}$ and $V$. 
higher amplitude transients. In the high-frequency case (480 $\mathrm{Hz}$ ), the decline in the output amplitude of the sinusoidal signal is much less, as expected from high pass filtering. Also, the output of the square signal resembles the input signal more because the signal alternates faster than the discharge rate of the capacitor formed by the human skin and touch screen insulator. The results depict that the stimuli on the mechanoreceptors have different waveform and amplitude than those of the input voltage signal.

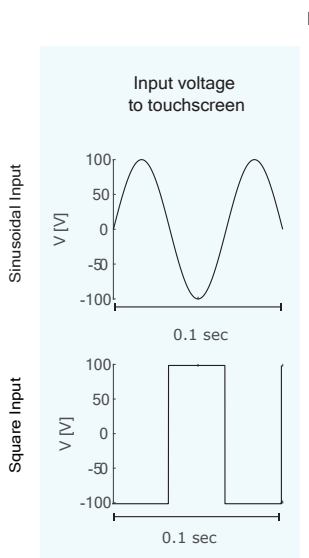

LOW FREQUENCY CASE $(15 \mathrm{~Hz})$
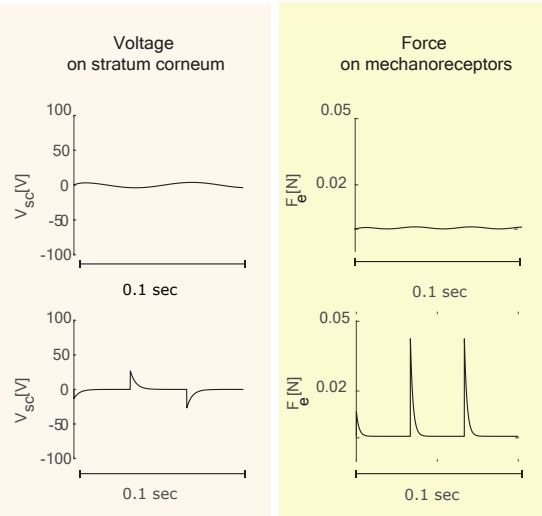

(a)

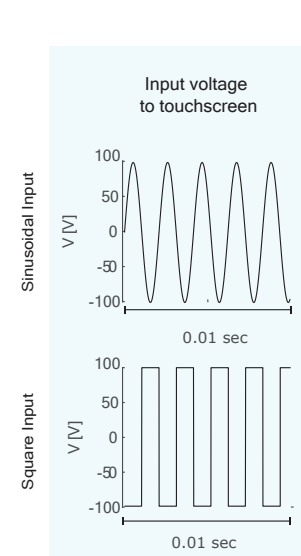

HIGH FREQUENCY CASE $(480 \mathrm{~Hz})$


(b)

Fig. 3. Simulation results: a. low frequency case, b. high frequency case.

If a complex waveform (containing many frequency components) arrives at mechanoreceptors, it can activate different psychophysical channels at different threshold levels [18], [21], [29]. These four psychophysical channels (NPI, NPII, NPIII, P) are mediated by four corresponding mechanoreceptor populations, which enable the tactile perception [18], [21], [22], [30], [31]. For this reason, the Fourier components of the stimulus should be weighted with the inverse of the human sensitivity curve to predict tactile sensitivity to complex stimuli [18]. The stimulus detection occurs at the channel where the maximum of this weighted function is located in the frequency domain. For example, a sinusoidal signal contains a single frequency component. To be able to detect this signal, its energy level must be higher than the human sensation threshold at that fre- quency. However, a square signal contains many frequency components. Detection occurs as soon as the energy level of one frequency component is higher than the human sensation threshold at that frequency. The tactile detection process for electrovibration is illustrated in Fig. 4. Here, a sinusoidal and a square voltage signals at the same fundamental frequency but different amplitude are applied to the touch screen. Due to electrical filtering of human finger, they generate electrostatic forces on the mechanoreceptors with the same amplitude. Therefore, the energy in $30 \mathrm{~Hz}$ component is the same for both force signals shown in Fig. 4c. However, the square wave input has higher frequency components, which are weighted more with respect to the human sensitivity curve (Fig. 4d). As a result, the weighted force signal contains a relatively high frequency component of $180 \mathrm{~Hz}$ (Fig. 4e). Therefore, in this illustration, the square wave is detected, but the sinusoidal wave is not.

\section{Materials and Methods}

\subsection{Experiment 1: Psychophysical Experiments}

To investigate how our detection threshold changes with input waveform, we conducted absolute detection experiments. These experiments enable us to determine the minimum voltage amplitude that the observer can barely detect [22], [31], [32], [33]. We aim to compare detection thresholds for sinusoidal and square wave voltage inputs at different frequencies to support our arguments made in Section 2.

\subsubsection{Participants}

We performed experiments with eight subjects (four female, four male) having an average age of 27.5 (SD: 1.19). All of the subjects were right-handed except one. All of them were engineering Ph.D. students. The subjects used the index finger of their dominant hand during the experiments. They washed their hands with soap and rinsed with water before the experiment. Also, their fingers and the touch screen were cleaned by alcohol before each measurement. The subjects read and signed the consent form before the experiments. The form was approved by Ethical Committee for Human Participants of Koç University.

\subsubsection{Stimuli}

We estimated absolute detection thresholds for seven input frequencies $(15,30,60,120,240,480$ and, $1920 \mathrm{~Hz})$ and two waveforms (sinusoidal and square).

\subsubsection{Experimental Setup}

The experimental setup used for the psychophysical experiment is shown in Fig. 5a. A touch screen (SCT3250, 3M Inc.) was placed on top of an LCD screen. An IR frame was placed above the touch screen to detect the finger location. The touch screen was excited with a voltage signal generated by a DAQ card (PCI-6025E, National Instruments Inc.) and augmented by an amplifier (E-413, PI Inc.). Subjects entered their responses through a computer monitor. An arm rest supported the subjects' arms during the experiments. For isolation of the background noises, subjects were asked to wear headphones displaying white noise during experiments. 


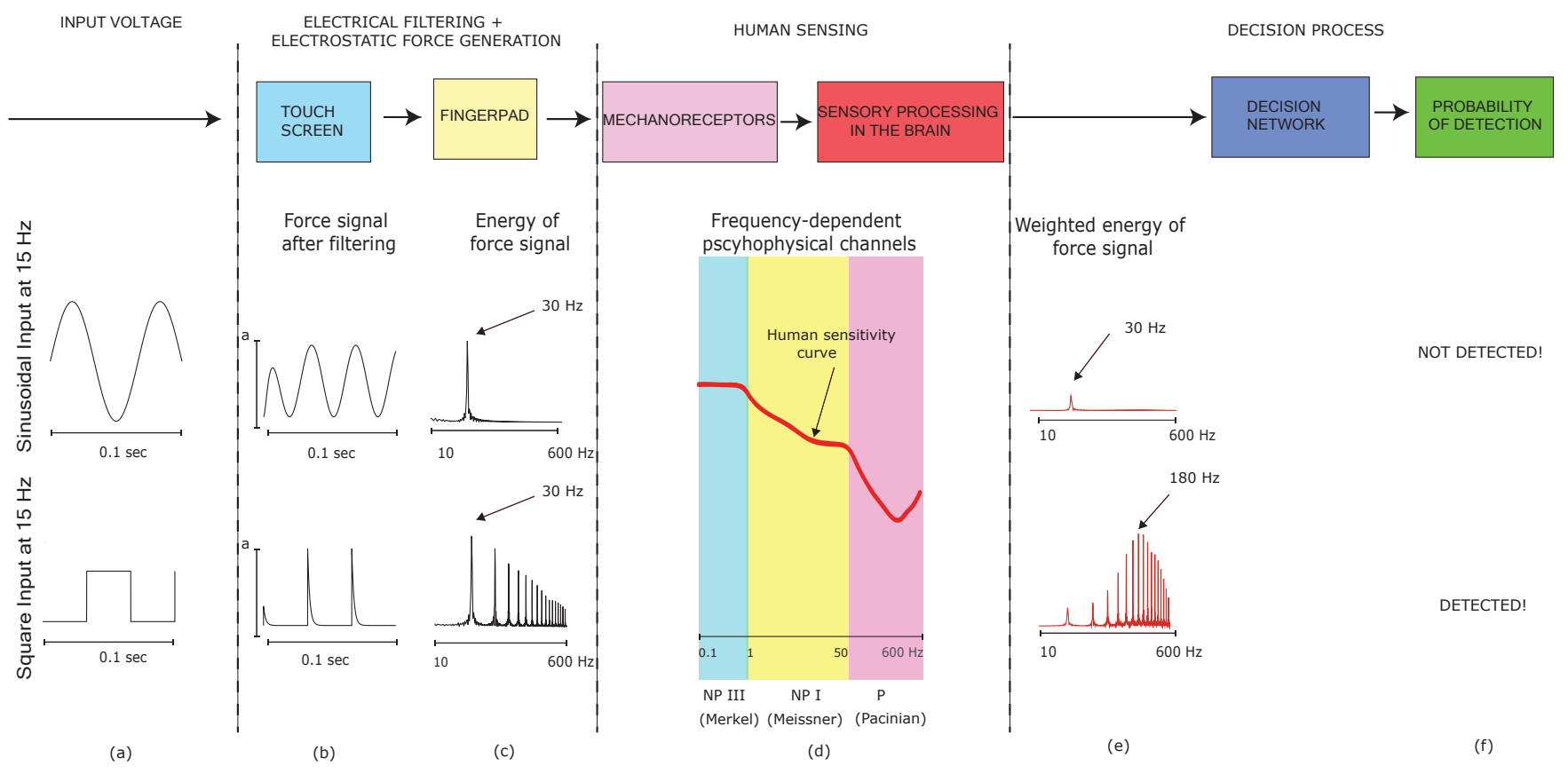

Fig. 4. An illustration of how tactile detection occurs. a. Input sinusoidal and square voltage signals at $15 \mathrm{~Hz}$ applied to touch screen at different amplitudes. b. These input signals are filtered electrically by human finger (see Fig. 3 for filtering process) before generating electrostatic forces with the same amplitude on the mechanoreceptors. c. The energy of the force signal originated from the sinusoidal wave contains only one frequency component $(30 \mathrm{~Hz}$ due to squaring in Equation 1) while the one from the square wave contains many frequency components. d. The frequencydependent human sensitivity curve; the most sensitive frequency regions of three psychophysical channels are color-coded. The fourth channel (NPII) does not appear in this illustration. e. When the Fourier components of the force signals are weighted by the inverse of the human sensitivity curve, the resulting signals from the sinusoidal and square waves have their maximum peaks at 30 and $180 \mathrm{~Hz}$, respectively. Moreover, the energy of the frequency component for the square wave case is larger than that of the sinusoidal one at those frequencies. $f$. Therefore, the square signal is detected, but the sinusoidal signal is not.

\subsubsection{Procedure}

We used the two-alternative-forced-choice method to determine the detection thresholds. This method enables criterion-free experimental results [22]. We displayed two regions (A and $\mathrm{B}$ ) on the $\mathrm{LCD}$ screen (Fig. 5a). Tactile stimulus was displayed in only one of the regions, and its location was randomized. The finger position of the subjects was detected via the IR frame. The subjects were asked to explore both areas consecutively and choose the one displaying a tactile stimulus.

We changed the amplitude of the tactile stimulus via one-up/two-down adaptive staircase method. This procedure decreases the duration of the experimentation by reducing the number of trials [22], [30], [31], [34], [35]. We started each session with the stimulus amplitude of $100 \mathrm{~V}$. This initial voltage amplitude provided sufficiently highintensity stimulus for all the subjects. The voltage amplitude of the new stimulus was adjusted adaptively based on the past responses of each subject. If the subject gave two consecutive correct answers, the voltage amplitude was decreased by $10 \mathrm{~V}$. If the subject had one incorrect response, the stimulus intensity was increased by $10 \mathrm{~V}$. The change of the response from correct to incorrect or the vice versa was counted as one reversal. After four reversals, the step size was decreased by $2 \mathrm{~V}$ to obtain a more precise threshold value, as suggested in [1]. We stopped the experiment after 18 reversals and estimated the absolute detection threshold as the average of the last 15 reversals (Fig. $5 b$ ). The subjects completed the experiments in 14 sessions, executed in 7 separate days (two sessions per day). The duration of each session was about 15-20 minutes.

\subsection{Experiment 2: Force \& Acceleration Measurements}

We measured the contact forces and accelerations acting on subjects' finger moving on the surface of the touch screen, which was actuated at the threshold voltages estimated in Experiment 1. Our main goal was to determine the frequency components of these recorded signals in order to validate our theoretical model and simulation results. We calculated the signal energies and weighted them with human sensitivity curve to estimate which components enabled the tactile detection. We also investigated the effect of scan speed on measured signals.

\subsubsection{Participants}

We conducted experiments with eight (four female and four male) subjects having the average age of 27.8 (SD: 2.1). The subjects read and signed the consent form before the experiments. The form was approved by Ethical Committee for Human Participants of Koç University. The subjects washed their hands with commercial soap and rinsed with water before each measurement. Then, they dried their hands in the room temperature and ambient pressure. Also, the touch screen was cleaned by alcohol before each measurement.

\subsubsection{Stimuli}

We measured accelerations and forces under 48 different conditions; there were 2 waveforms (sinusoidal, square), 


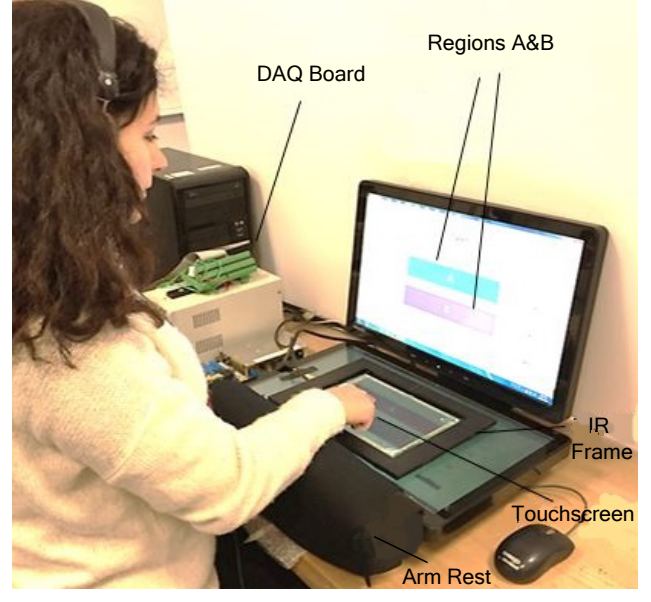

(a)

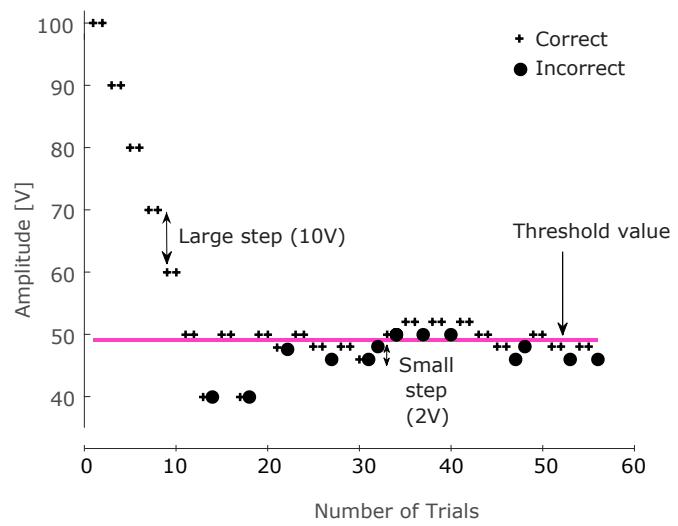

(b)

Fig. 5. a. Experimental setup used in our psychophysical experiments. b. An example data set collected by one up-two down adaptive staircase method.

6 frequencies $(15,30,60,120,240,480 \mathrm{~Hz})$, and 4 finger scan speeds $(10,20,50,100 \mathrm{~mm} / \mathrm{s})$, which are tabulated in Table 2. In each measurement, one parameter was changed while fixing the others. We selected the finger scan speeds based on the values used in the earlier studies [36], [37], [38], [39]. The amplitude of the input signals was chosen $8 \mathrm{~dB}$ SL (sensation level: $8 \mathrm{~dB}$ higher than the threshold) more than the averaged threshold values measured in Experiment 1 (see Section 3.1).

Initially, we performed two separate control measurements to test the reliability of the collected data ${ }^{2}$. First, the forces and accelerations were measured when the finger was stationary in 12 conditions to observe the electromagnetic interference (EMI) effect on the sensors (Table 2). Second, the forces and accelerations were measured without any electrostatic excitation in 4 conditions (Table 2). Therefore, 64 different (48 test, 16 control) measurements were performed in total for each subject.

2. In the first set of control measurements, we checked the signal to noise ratio (SNR). If the SNR value of a measurement was lower than $5 \mathrm{~dB}$, that measurement was repeated. In the second set of control measurements, we checked the signal energies due to finger motion without any electrostatic excitation. These energies were compared to those obtained from the test measurements to investigate the effect of electrostatic excitation (see Section 4.2).
TABLE 2

Experimental Parameters.

\begin{tabular}{|c|c|c|c|}
\hline Type & Parameter & Value & Unit \\
\hline \hline \multirow{3}{*}{ Test } & Frequency & $15,30,60,120,240,480$ & $\mathrm{~Hz}$ \\
\cline { 2 - 4 } & Waveform & Sinusoidal, Square & - \\
\cline { 2 - 4 } & Scan Speed & $10,20,50,100$ & $\mathrm{~mm} / \mathrm{s}$ \\
\hline \hline $\begin{array}{c}\text { Control 1 } \\
\text { EMI Effect) }\end{array}$ & Frequency & $15,30,60,120,240,480$ & $\mathrm{~Hz}$ \\
\cline { 2 - 4 } & Waveform & Sinusoidal, Square & - \\
\hline $\begin{array}{c}\text { Control 2 } \\
\text { (No excitation) }\end{array}$ & Scan Speed & $10,20,50,100$ & $\mathrm{~mm} / \mathrm{s}$ \\
\hline
\end{tabular}

\subsubsection{Experimental Setup}

The experimental setup was similar to the one used in our psychophysical experiments (Fig. 5a). For this experiment, the touch screen (SCT3250, 3M Inc.) was placed on top of a force sensor (Nano17, ATI Inc.). The sensor was attached to the screen and an aluminium base using double-sided adhesive tapes (3M Inc.). The aluminum base was also attached to a stationary table by the same adhesive tape. The touch screen was excited with a voltage signal generated by a signal generator (33220A, Agilent Technologies Inc.). The voltage signal from the generator was amplified by an amplifier (E-413, PI Inc.) before transmitted the touch screen. An IR frame was placed on top of the touch screen to measure the finger scan speed during experiments. An accelerometer (ADXL 335, Analog Devices Inc.) was glued on the fingernail of the subjects. The accelerometer and force data were acquired by two separate DAQ cards (USB-6251 and PCI-6025E, NI Inc.). The cables of the accelerometer were taped on the finger and arm of the subjects as shown in Fig. 6. Both accelerometer and force data were acquired using LabView (NI, Inc.). An arm rest was used to support the subjects' arm during the experiments. The subjects were asked to wear a ground strap on their stationary wrist. The subjects were also asked to synchronize their scan speeds with the speed of a visual cursor displayed on the computer screen.

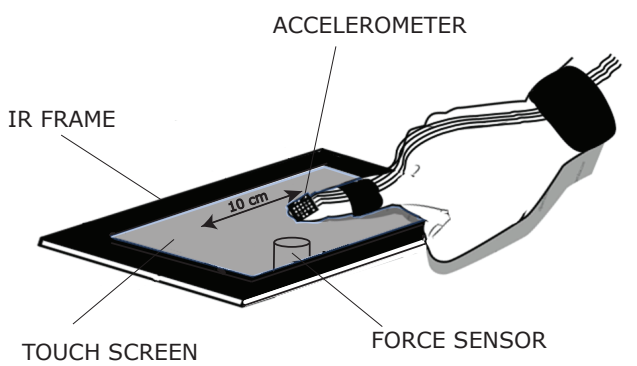

Fig. 6. Illustration for the attachment of force sensor and accelerometer.

\subsubsection{Procedure}

The subjects were instructed to sit on a chair in front of the experimental setup and move their index fingers back and forth in the horizontal direction on the touch screen. They were asked to move their finger only in a $10 \times 3 \mathrm{~cm}$ rectangular region on the touch screen. They were asked to synchronize their fingers with the motion of a moving 
cursor on the computer screen. Also, they received visual feedback about the magnitude of the normal force that they applied to the touch screen. For this purpose, two led lights were displayed on the computer screen and used to keep the normal force between 0.1 and $0.6 \mathrm{~N}$. We selected this range based on the normal forces reported in the literature as relevant to tactile exploration [39], [40]. If the user applied less than $0.1 \mathrm{~N}$ to the touch screen, the led labelled as "press more" turned to green. However, if the user applied more than $0.6 \mathrm{~N}$, the led labelled as "press less" turned to red. The subjects were instructed to complete four strokes (two forward, two backward) under each experimental condition.

Before starting the experiment, the subjects were given instructions about the experiment, and asked to complete a training session. This training session enabled subjects to adjust their finger scan speed and normal force before the actual experimentation. The experiments were performed in two blocks. The first and second blocks had six and seven sessions respectively. The experimental blocks were formed based on the input voltage waveform whereas the sessions were based on the input voltage frequency. The second block also contained one session without any input voltage. It took approximately 1.5 hours to complete all the measurements for a subject, including the time for attaching the accelerometer to the subjects' finger and the training session.



Fig. 7. Data collected during one experimental session. The input voltage was a square wave at $60 \mathrm{~Hz}$.

\subsubsection{Data Analysis}

The force and acceleration data were analyzed in Matlab. An example data collected during one session is shown in Fig. 7. The figure shows force and acceleration data recorded at different scan speeds. We calculated the displacement values by integrating the acceleration data twice as suggested in [41].
The collected force, acceleration and displacement data were segmented according to the finger scan speed (see coloured regions in Fig. 7). Then, DC offset was removed from each segment by subtracting the mean values. To remove the low-frequency noise due to finger motion, data in each stroke was filtered by a high-pass filter having a cutoff frequency of $10 \mathrm{~Hz}$. Afterwards, the RMS of each stroke was calculated and an average RMS was obtained for each finger speed using the data of 4 strokes.

For detection analysis, power spectrum of each stroke was calculated for the signals in the normal direction. Then, an average power spectrum was obtained for each finger speed using the power spectrum of 4 strokes. The peak frequencies were determined using this spectrum. The energy (in unit time) of each peak frequency was calculated by integrating its power spectrum data for the peak interval. Finally, the calculated raw energies were multiplied by the inverse of the normalized human sensitivity function to obtain the weighted ones (Fig. 8). We used the human sensitivity functions reported in [42], [43] for the force, acceleration and displacement data, respectively. Moreover, we calculated the corresponding electrostatic forces generated by the same waveforms and amplitudes via Matlab simulations. We also calculated the weighted energies of those simulated forces using the same data analysis approach discussed above.

In addition, the average friction coefficient was calculated by dividing the unfiltered lateral force of each stroke to those of normal force. Then, an average friction coefficient of each condition was obtained using the data of 4 strokes.
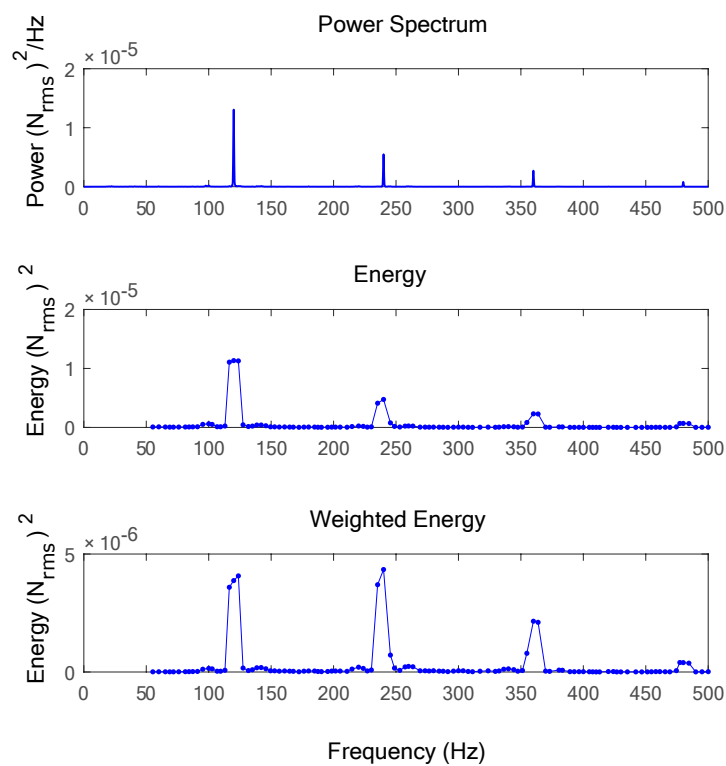

Fig. 8. Exemplar plots of average power spectrum, energy (in unit time), and weighted energy as a function of frequency. The plots were generated using the force data recorded at the finger scan speed of 20 $\mathrm{mm} / \mathrm{s}$ (the input voltage was a square wave at $60 \mathrm{~Hz}$ ).

\section{Results}

\subsection{Results of Experiment 1}

Fig. 9 depicts the measured threshold voltages for seven fundamental frequencies $(15,30,60,120,240,480,1920 \mathrm{~Hz})$ and two different waveforms (sinusoidal and square). 
We analyzed the results using two-way analysis of variance (ANOVA) with repeated measures. Both main effects (frequency and waveform) were statistically significant on the threshold levels $(\mathrm{p}<0.01)$. Moreover, there was a statistically significant interaction between frequency and waveform $(\mathrm{p}<0.01)$.

Additionally, the effect of the waveform on our tactile perception at each frequency was analyzed by Bonferroni corrected paired $\mathrm{t}$-tests. The results showed that there was a statistically significant effect of the waveform on our haptic perception for fundamental frequencies less than $60 \mathrm{~Hz}$. The difference between square and sinusoidal waves was significant at frequencies greater than and equal to $60 \mathrm{~Hz}$. The corrected p-values for each frequency $(15,30,60,120$, $240,480,1920 \mathrm{~Hz}$ ) are $0.008,0.016,1,1,1,0.168$, and 0.128 , respectively.



Fig. 9. The average detection thresholds of the subjects for seven fundamental frequencies $(15,30,60,120,240,480,1920 \mathrm{~Hz})$ and two different waveforms (sinusoidal and square).

\subsection{Results of Experiment 2}

The RMS values calculated for each condition from acceleration and force data (lateral and normal), and friction coefficients are plotted against fundamental frequencies of the input signals (Fig. 10). The data from different scan speeds were averaged for the clarity of plots. The results were analysed using three-way analysis of variance (ANOVA) with repeated measures. The results showed that finger scan speed had a significant effect on force, acceleration, and friction coefficient $(\mathrm{p}<0.05)$.

To test the reliability of the measurement results, the average energies calculated for no electrostatic excitation were compared to those of electrostatic excitation using independent t-tests. Electrovibration generated a statistically significant difference in all calculated energies for both waveforms (sinusoidal and square) and for each response type (acceleration, force, and displacement) $(\mathrm{p}<0.05)$.

The average weighted energies calculated for each actuated condition from displacement, acceleration and force data (normal) are plotted against fundamental frequencies of the input signal (Figs. 11a-c). They are also plotted as a function of the frequency component having the highest energy (Figs. 11e-g). The frequency interval in which the Pacinian channel is the most sensitive is marked as pink. Moreover, the average weighted energies estimated from
Matlab simulations are also compared to those of the experimental results (Figs. 11d and h).

We analyzed the weighted energy results for all the measured variables using three-way analysis of variance (ANOVA) with repeated measures. The effects of waveform, frequency, and scan speed on the weighted energy were significant $(p<0.05)$. Their interactions except the one between speed and frequency were also statistically significant $(\mathrm{p}<0.05)$. Moreover, Bonferroni corrected paired $\mathrm{t}$ tests showed that the weighted energies were statistically different for sinusoidal and square waves at fundamental frequencies 15, 30 and $480 \mathrm{~Hz}(\mathrm{p}<0.05)$, and similar for the other frequencies.

For square signals, we calculated the proportion of the frequency components that were within the sensitivity range of the Pacinian channel $(100-500 \mathrm{~Hz})$ to the total number of components for each response type (acceleration, force, and displacement) for measured and simulated variables. The results showed that the frequency components having the highest energies were accumulated between 100$500 \mathrm{~Hz}$ for the square signals.

\section{Discussion}

Our results showed that human perception of electrovibration on touch screens is frequency-dependent as in vibrotactile studies. The detection thresholds obtained from our psychophysical experiments (Fig. 9) followed the well known U-shaped human sensitivity curve. The threshold values were low between $60 \mathrm{~Hz}$ and $240 \mathrm{~Hz}$, and higher for the rest. The corresponding detection energies of force (measured and simulated), acceleration (measured) and displacement (measured) signals calculated at these thresholds naturally displayed an inverted U-shape trend as a function of frequency (Figs. 11a-d). These results are consistent with the existing vibrotactile literature [18], [21], [22], [31], [43]. In earlier studies, the detection thresholds of the index or middle finger were measured as a function of frequency by using various contactors. Typically, sinusoidal displacements with slow onset and offset times was used as stimuli, which generate mechanical excitation with a single frequency component. In our case, alternating electrostatic forces are generated at the contact interface based on the square of the voltage applied to the touch screen (Equation 1). This nonlinear transformation introduces frequency components not present in the original signal. For example, when a pure sinusoidal voltage is applied to the touch screen, the force waveform has twice the frequency of the input wave. Hence, the detection results presented in Fig. 9 for square and sinusoidal stimuli should be interpreted by multiplying the values on the frequency axis with a factor of two. When the calculated energies are plotted against the frequency component having the highest energy (Fig. 11 eh), the peak values are between 100 and $500 \mathrm{~Hz}$, which is similar to those reported in the earlier vibrotactile literature [18], [22], [43]. In [1], Bau et al. measured absolute detection thresholds of electrovibration stimuli for sinusoidal inputs. Their results also followed a U-shaped trend, but their detection threshold values for sinusoidal inputs were slightly lower than our results. This difference might be caused by the experimental factors such as the angle of contact, 


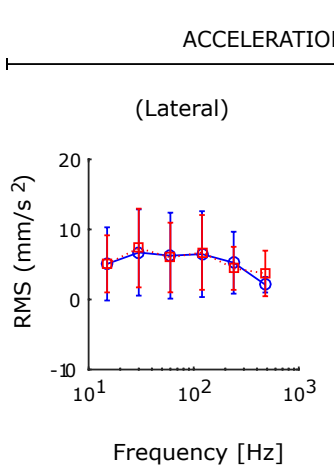

(a)

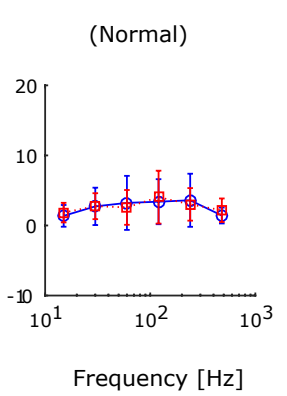

(b)

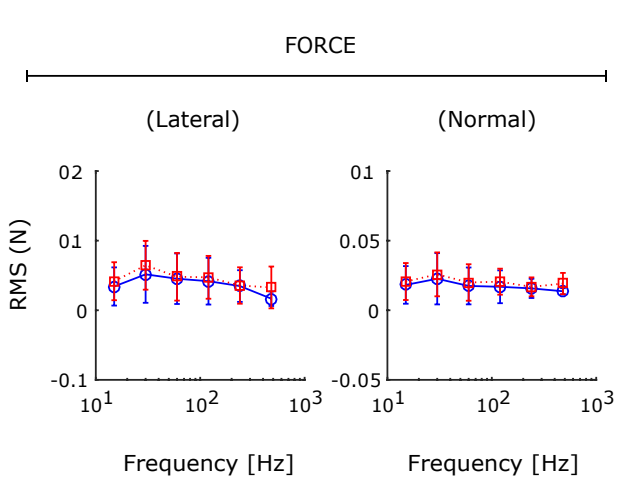

(c)
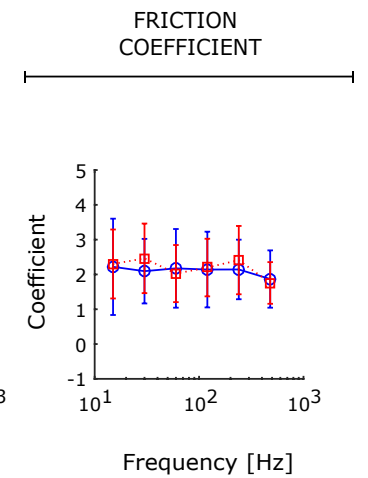

(e)

$\rightarrow \sin$

$\cdots$.

Fig. 10. The means and standard deviations of acceleration (a-b), force (c-d), and friction coefficient (e). The data from different scan speeds were averaged for the clarity of plots.

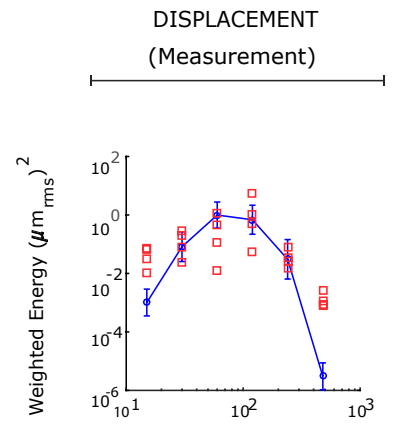

(a)

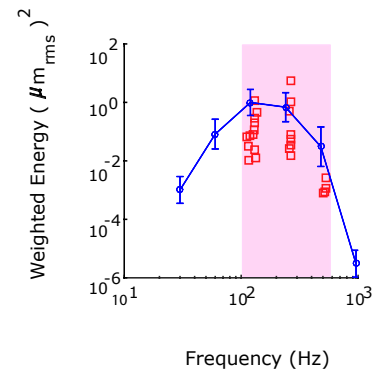

(e)

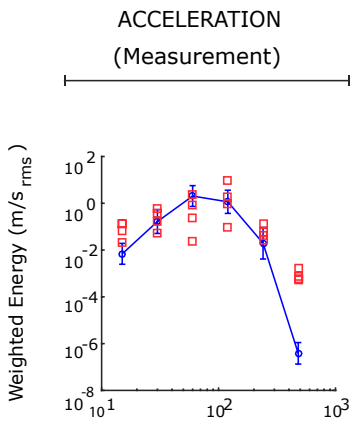

(b)

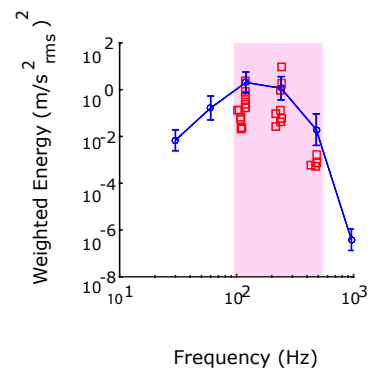

(f)

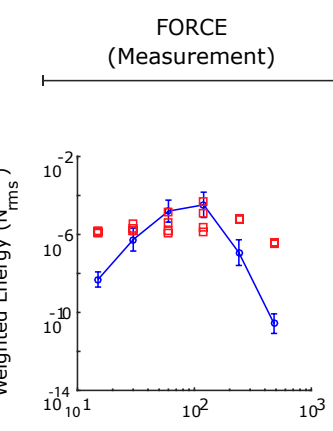

(c)

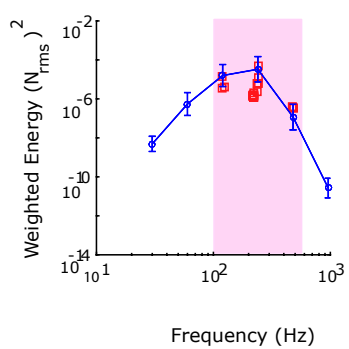

(g)
FORCE

(Simulation \& Measurement)



(d)

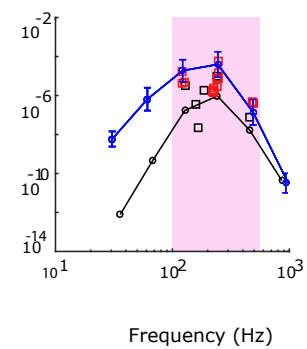

(h)

$\rightarrow \sin , \exp \quad \rightarrow \sin , \operatorname{sim}$

a squ, exp

a squ, sim

Fig. 11. The weighted energies of the displacement, acceleration, and force signals at threshold are plotted against the input fundamental frequency $(\mathrm{a}-\mathrm{c})$ and the frequency component with the highest energy $(\mathrm{e}-\mathrm{g})$. The output data for the input sinusoidal signals recorded at different scan speeds were averaged for the clarity of plots. The output data for the input square signals were not averaged because they contain energy components at many frequencies. The weighted energies of the simulated force signals at threshold are plotted against the fundamental frequency (d) and the frequency component with the highest energy $(\mathrm{h})$ and compared with those of the measurements. The pink regions indicate the frequency interval where the Pacinian channel is the most sensitive.

movement direction, environmental factors such as finger moisture and contact temperature, the number of subjects, and subject-to-subject variability such as the variability in fingerprints and finger electromechanical properties [39], [40], [44], [45], [46], [47].

We found that participants were more sensitive to square excitation than sinusoidal one for frequencies lower than $60 \mathrm{~Hz}$. The results suggested that Pacinian channel was the primary psychophysical channel in the detection of the electrovibration stimuli caused by all the square-wave inputs tested in this study. If a complex waveform, i.e. one which has many frequency components, is applied to the touch screen, the frequency components in the range of 50$250 \mathrm{~Hz}$ would be mostly active in stimuli detection due to the high sensitivity of Pacinian channel at twice of these frequencies. For example, due to electrical filtering of finger, low-frequency components of a square wave excitation are suppressed. Therefore, the voltage across the dielectric layer contains exponentially decaying high-frequency transients. The electrostatic force generated based on these transients 
is rather complex, including twice the frequencies and distortion products of the input signal components. Due to the frequency-dependent human tactile sensitivity, the frequency components in the force waveform will not be equally effective in detection (see Fig. 4). For example, when the weighted energies are plotted as a function of fundamental frequencies (Fig. $11 \mathrm{a}-\mathrm{d}$ ), it is difficult to interpret the results in terms of tactile detection. On the other hand, when the weighted energies are plotted as a function of frequency components with the highest energies in the force, acceleration, and displacement signals in our study (Fig. 11 $\mathrm{e}-\mathrm{h}$ ), the peak values fell into the range of $100-500 \mathrm{~Hz}$ (see the pink regions in Fig. $11 \mathrm{e}-\mathrm{h}$ ), which suggest that mainly the Pacinian channel was effective in detection for square wave inputs [18], [21].

In Matlab simulations, we used the values of the human skin parameters $\left(\rho_{s c}\right.$ and $\left.\epsilon_{s c}\right)$ measured at the fundamental frequencies. Although this is a valid assumption for the sinusoidal wave, it is a simplification for the square wave, since square wave contains many frequency components. This limitation might have contributed to the differences in experimental and the simulation results. In general the force amplitudes and energies estimated through simulations were lower than those measured through experiments for both square and sinusoidal waves (Fig. $11 \mathrm{~d}, \mathrm{~h}$ ). Experimental factors such as moisture, temperature, and subjectto-subject variability of fingertip mechanical and electrical properties might have contributed to the differences [39], [40], [44], [45], [46], [47]. For example, measuring electrical impedances directly from the subjects might lead to a better match of the experimental and simulation results. Also, future models of mechanical interpretation of electrovibration may help to explain the mismatch. For example, a more accurate estimation of the force energies at the mechanoreceptor level could potentially be obtained by linking the electrostatic forces generated at the fingerpad to the mechanical forces measured at the contact interface during finger movement.

The changes in RMS of measured mechanical forces, accelerations and friction coefficients as a function of waveform were not significant most probably because the input signals were normalized referenced to the threshold levels. However, when we inspect Fig. 10, the RMS values as a function of frequency are almost constant. This has to be due to the nature of RMS measurement which is not suitable for modelling the detection. On the other hand, it simplifies the illustration of time varying sensor output data.

Measured force, acceleration and friction coefficients were affected by finger scan speed in a complex manner suggesting that it might also affect our haptic perception accordingly. The results showed that the magnitude of contact forces and accelerations were appeared to be positively correlated with the scan speed though the friction showed a negative correlation. Similar results were also obtained in the earlier studies. Using an artificial finger which had similar electrical and mechanical properties of a real human finger, Mullenbach et. al. investigated that lateral forces generated by electrovibration increased as a function of scan speed [48]. Moreover, in our experiments the acceleration and force energies increased as the scan speed was increased. The earlier studies in tribology literature support this result [44], [49], [50], [51], [52]. The effect of scan speed on the measured forces and accelerations and their energies suggest that the viscoelastic characteristics of human finger also plays a role in tactile sensing of electrovibration. The possible effect of skin mechanics on psychophysical detection thresholds were also suggested by Yildiz and Güçlü in [31]. In that study, they measured vibrotactile detection thresholds of Pacinian channel at $250 \mathrm{~Hz}$ and mechanical impedances of fingertips of seven subjects. They reported that there was a significant positive correlation between loss moduli of the skin and detection thresholds.

As far as we know, this is the first study which investigates the effect of input voltage waveform on haptic perception of electrovibration in the frequency domain. The earlier research studies have already investigated the detectability and discriminability of mechanical waveforms in real and virtual environments and the results of these studies can be compared with ours. For example, Summers et al. [53], observed that vibrotactile sine waves and monophasic/tetraphasic pulses at suprathreshold levels resulted in similar scores in a frequency identification task. They concluded that temporal cues are more important than spatial cues in that particular task. We think their results can be interpreted that the strongest frequency component in complex waveforms (after correction for human sensitivity) drives the stimulus detection. Cholewiak et al. [54] investigated the perception of virtual gratings containing multiple spectral components. They performed detection and discrimination experiments with virtual sinusoidal and square gratings displayed by a force-feedback device at various spatial frequencies. Their results showed that detection thresholds of square gratings were lower than the sinusoidal ones at lower spatial frequencies. Similar to our results, they explained that the square gratings are detected based on their harmonic components having the lowest detection threshold.

\section{CONCLUSION}

In this study, we investigated how input voltage waveform affects our haptic perception of electrovibration on touch screens. Through psychophysical experiments with eight subjects, we first measured the detection thresholds of electrovibration stimuli generated by sinusoidal and square voltages at various frequencies. We observed that the subjects were more sensitive to square wave stimuli than sinusoidal one for fundamental frequencies lower than $60 \mathrm{~Hz}$. We hypothesized that the sensation difference of waveforms in low fundamental frequencies is due to frequency-dependent electrical properties of human skin and human tactile sensitivity. To validate our hypothesis and observe if there was any other physical factor which may affect our perception of electrovibration perception, we conducted a second experiment with another group of eight subjects. We collected force and acceleration data from fingertips of the subjects while they explored a touch screen displaying electrovibration stimuli at threshold voltages. We analyzed the collected data in frequency domain by taking the human tactile sensitivity curves given in [42], [43] into account. The results suggested that Pacinian was the primary psychophysical channel in the detection of the 
square wave input signals tested in this study. Moreover, our results showed that measured acceleration and force data are affected by finger scan speed.

To the best of our knowledge, this is the first detailed study investigating the effect of input voltage waveform on haptic perception of electrovibration. Our findings not only help us to understand the mechanism of human tactile sensing of electrovibration but also may help engineers and designers to develop applications displaying tactile effects to the users through a touch screen. For example, a user interface developer designing a virtual dial on a touch screen may prefer to use low frequency square pulses rather than sinusoidal ones to display tactile dents. On the other hand, less detectable sinusoidal signals could be used to display frictional feedback to the user while she/he turns the dial on the screen for better control. Furthermore, the perception difference between waveforms may also be used for pattern and edge recognition. When a blind user explores a virtual shape on a touch screen, the edges can be conveyed by low-frequency square waves while a sinusoidal wave can be used for smoother feeling inside. Moreover, since the detection of tactile stimuli is determined by frequency components below $1 \mathrm{kHz}$, it may not be necessary to transmit higher frequency components which would be lower than the detection thresholds. This ensures transmission of less data without sacrificing the perceptual needs for systems with limited bandwidth.

Furthermore, the results of this study can be a guide for developing an electromechanical model of human finger linking the electrostatic force displayed to human finger pad by electrovibration to the mechanical forces felt at the finger contact interface. As our results suggest, frictional forces modulated by the contact interface and scan speed have influence on mechanical vibrations measured at fingertip and hence potentially on our tactile perception. Finally, our results also suggest that tactile perception of electrovibration is similar to that of vibrotactile stimuli. We have recently started to investigate psychophysics of masking by electrovibration as done similarly in vibrotactile studies. This may help us to augment tactile effects displayed to the user through the touch screen.

\section{ACKNOWLEDGMENTS}

The Scientific and Technological Research Council of Turkey (TUBITAK) supported this work under Student Fellowship Program BIDEB-2211. Also, the authors would like to thank to Prof. Dr. Edward Colgate for his valuable comments. Moreover, Y.V thanks Gokhan Serhat and Ozan Caldiran for their fruitful discussions during this study.

\section{REFERENCES}

[1] O. Bau, I. Poupyrev, A. Israr, and C. Harrison, "Teslatouch: Electrovibration for touch surfaces," in Proc. of the 23nd annual ACM symposium on User interface software and technology (UIST'10), NewYork, USA, 2010, pp. 283-292.

[2] J. Linjama and V. Mkinen, "E-sense screen: Novel haptic display with capacitive electrosensory interface." in Proc. 4th Workshop for Haptic and Audio Interaction Design (HAID'09), Dresden, Germany, 2009.

[3] S. Kim, A. Israr, and I. Poupyrev, "Tactile rendering of $3 \mathrm{~d}$ features on touch surfaces." St. Andrews: UIST'13, 2013.
[4] D. Wijekoon, M. Cecchinato, E. Hoggan, and J. Linjama, "Electrostatic modulated friction as tactile feedback: Intensity perception," in Haptics: Perception, Devices, Mobility, and Communication Lecture Notes in Computer Science, 2012, vol. 7282, pp. 613-624.

[5] Y. Vardar, B. Güçlü, and C. Basdogan, "Effect of waveform in haptic perception of electrovibration on touchscreens," in Haptics: Perception, Devices, Control, and Applications: 10th International Conference, EuroHaptics 2016, London, UK, July 4-7, 2016, Proceedings, Part I, 2016.

[6] A. Johnsen and K. Rahbek, "A physical phenomenon and its applications to telegraphy, telephony, etc." IEE Journal, vol. 61, pp. 713-725, 1923.

[7] E. Mallinckrodt, A. Hughes, and W. Sleator, "Perception by the skin of electrically induced vibrations," Science, vol. 118, pp. 277$278,1953$.

[8] S. Grimnes, "Electrovibration, cutaneous sensation of microampere current," Acta Physiol Scand, vol. 118, pp. 1565-1574, 1983.

[9] R. Strong and D. Troxel, "An electrotactile display," IEEE Transactions on Man-Machine Systems, vol. 11, no. 1, pp. 72-79, 1970.

[10] D. Beebe, C. Heymel, K. Kaczmarek, and M. Tyler, "A polyimideon-silicon electrostatic fingertip tactile display," in Proc. of the IEEE 17th Annual Conference on Engineering in Medicine and Biology Society, Montreal, Que, 1995, pp. 1545-1546.

[11] H. Tang and D. Beebe, "A microfabricated electrostatic haptic display for persons with visual impairments," IEEE Transactions on Rehabilitation Engineering, vol. 6, no. 3, pp. 241-248, 1998.

[12] A. K. Agarwal, K. Namni, K. A. Kaczmarek, M. E. Tyler, and D. J. Beebe, "A hybrid natural/artificial electrostatic actuator for tactile stimulation," in Proc. of the 2nd Annual Conference on Microtechnologies in Medicine and Biology, Madison, Wisonsin USA, 2002, pp. 341-345.

[13] K. Kaczmarek, K. Nammi, A. Agarwal, M. Tyler, S. Haase, and D. Beebe, "Polarity effect in electrovibration for tactile display," IEEE Transactions on Biomedical Engineering, vol. 53, no. 10, pp. 2047-2054, 2006

[14] D. Mayer, M. Peshkin, and E. Colgate, "Fingertip electrostatic modulation due to electrostatic attraction," in Proc. IEEE World Haptics Conference (WHC'13), Daejeon, South Korea, Apr. 2013, pp. $43-48$.

[15] E. Vezzoli, M. Amberg, F. Giraud, and B. Lemaire-Semail, "Electrovibration modeling analysis," in Proc. of the 9th International Conference, Eurohaptics 2014, Versailles, France, 2014, pp. 369-376.

[16] T. Yamamoto and Y. Yamamoto, "Dielectric constant and resistivity of epidermal stratum corneum," Medical and Biological Engineering, pp. 494-500, 1976.

[17] H. Kim, J. Kang, K. Kim, K. Lim, and J. Ryu, "Method for providing electrovibration with uniformed density," IEEE Transactions on Haptics, vol. 8, no. 4, pp. 492-496, 2015.

[18] G. A. Gescheider, S. J. Bolanowski, J. V. Pope, and R. T. Verrillo, "A four-channel analysis of the tactile sensitivity of the fingertip: frequency selectivity, spatial summation, and temporal summation," Somatosensory and Motor Research, vol. 19, no. 2, pp. 114-124, 2002

[19] K. R. Demarest, Engineering Electromagnetics. Prentice Hall, 1998.

[20] C. D. Shultz, M. Peshkin, and E. Colgate, "Surface haptics via electroadhesion: Expanding electrovibration by johnsen and rahbek," in Proc. IEEE World Haptics Conference (WHC'15), Evanston, USA, Jun. 2013, pp. 57-62.

[21] S. Bolanowski, G. Gescheider, R. T. Verrillo, and C. Checkosky, "Four channels mediate the mechanical aspects of touch," Acoustic Society of America, vol. 84, no. 5, pp. 1680-1694, 1988.

[22] B. Güçlü and C. Öztek, "Tactile sensitivity of children: Effects of frequency, masking, and the non-pacinian I psychophysical channel." Journal of Experimental Child Psychology, vol. 98, pp. 113$130,2007$.

[23] M. Yıldız, İ. Toker, F. Özkan, and B. Güçlü, "Effects of passive and active movement on vibrotactile detection thresholds of the pacinian channel and forward masking," Somatosensory \& Motor Research, vol. 32, no. 4, pp. 262-272, 2015.

[24] B. Güçlü, S. Bolanowski, and L. Pawson, "End-to-end linkage (eel) clustering algorithm: A study on the distribution of meissner corpuscles in the skin," Journal of Computational Neuroscience, vol. 15, pp. 19-28, 2003.

[25] B. Güçlü, G. Mahoney, L. Pawson, R. Smith, and S. Bolanowski, "Localization of merkel cells in the skin: An anatomical model." Somatosensory \& Motor Research, vol. 25, pp. 123-138, 2008.

[26] B. Güçlü, E. Schepis, S. Yelke, C. Yücesoy, and B. S.J., "Ovoid geometry of the pacinian corpuscle is not the determining factor 
for mechanical excitation." Somatosensory \& Motor Research, vol. 23, pp. 119-126, 2006.

[27] S. Grimnes, "Skin impedance and electro-osmosis in the human epidermis," Medical $\mathcal{E}$ Biological Engineering $\mathcal{E}$ Computing, vol. 21, pp. 739-749, 1983.

[28] K. Kaczmarek, J. Webster, P. Rita, and W. Tompkins, "Electrotactile and vibrotactile displays for sensory substitution systems," IEEE Transactions on Biomedical Engineering, vol. 38, no. 1, 1991.

[29] G. Aiello, "Multidimensional electrocutaneous stimulation," IEEE Transactions on Rehabilitation Engineering, vol. 6, no. 1, 1998.

[30] B. Güçlü and S. Bolanowski, "Frequency responses of cat rapidly adapting mechanoreceptive fibers," Somatosensory \& Motor Research, vol. 20, pp. 249-263, 2003.

[31] M. Yıldız and B. Güçlü, "Relationship between vibrotactile detection threshold in the pacinian channel and complex mechanical modulus of the human glabrous skin." Somatosensory \& Motor Research, vol. 30, pp. 37-47, 2013.

[32] W. H. Ehrenstein and A. Ehrenstein, "Psychophysical methods," in Modern Techniques in Neuroscience Research. Springer Berlin Heidelberg, 1999, pp. 1211-1241.

[33] B. Güçlü and S. Bolanowski, "Vibrotactile thresholds of the nonpacinian I channel: I. methodological issues," Somatosensory \& Motor Research, vol. 22, pp. 49-56, 2005.

[34] H. Levitt, "Transformed up-down methods psychoacoustics," The Journal of Acoustical Society of America, vol. 49, no. 2, pp. 467-477, 1971

[35] M. R. Leek, "Adaptive procedures in psychophysical research," Perception and Psychophysics, vol. 63, no. 8, pp. 1279-1292, 2001.

[36] R. Fagiani and M. Barbieri, "Modelling of finger-surface contact dynamics," Tribology International, vol. 74, pp. 130-137, 2014.

[37] M. Wiertlevski and V. Hayward, "Mechanical behavior of the fingertip in the range of frequencies and displacements relevant to touch." Journal of Biomechanics, vol. 45, pp. 1869-1874, 2012.

[38] T. Yoshioka, J. Craig, G. Beck, and S. Hsiao, "Perceptual constancy of texture roughness in the tactile system," The Journal of Neuroscience, vol. 31, no. 48, p. 1760317611, 2007.

[39] M. Adams, S. Johnson, P. Lefèvre, V. Lévesque, V. Hayward T. André, and J. Thonnard, "Finger pad friction and its role in grip and touch," Journal of The Royal Society Interface, vol. 10, no. 80, 2012.

[40] B. Delhaye, P. Lefevre, and J.-L. Thonnard, "Dynamics of fingertip contact during the onset of tangential slip." Journal of the Royal Society Interface, vol. 11, 2014

[41] G. A. Gescheider, J. Wright, and R. Verrillo, Information-processing channels in the tactile sensory system a psychophysical and psysiological analysis. Psychology Press, 2009.

[42] C. Hatzfeld, S. Cao, M. Kupnik, and R. Werthschtzky, "Vibrotactile force perception - absolute and differential thresholds and external influences," IEEE Transactions on Haptics, vol. 9, no. 4, pp. 586-597, 2016.

[43] M. Morioka and M. Griffin, "Thresholds for the perception of hand-transmitted vibration: Dependence on contact area and contact location," Somatosensory and Motor Research, vol. 22, no. 4, pp 281-297, 2005.

[44] S. M. Pasumarty, S. A. Johnson, S. A. Watson, and M. J. Adams, "Friction of the human finger pad: influence of moisture, occlusion and velocity," Tribology Letters, vol. 44, pp. 117-127, 2011.

[45] S. Derler and L. Gerhardt, "Tribology of skin: review and analysis of experimental results for the friction coefficient of human skin," Tribology Letters, vol. 45, pp. 1-27, 2012.

[46] T. Andre, V. Levesque, V. Hayward, P. Lefevre, and J. Thonnard, "Effect of skin hydration on the dynamics of fingertip gripping contact," Journal of Royal Society Interface, vol. 8, pp. 1574-1583, 2011.

[47] S. Derler, L.-C. Gerhardt, A. Lenz, E. Bertaux, and M. Hadad, "Friction of human skin against smooth and rough glass as a function of the contact pressure," Tribology International, vol. 42, pp. 1565-1574, 2009.

[48] J. Mullenbach, M. Peshkin, and E. Colgate, "eshiver: Force feedback on fingertips through oscillatory motion of an electroadhesive surface," in Haptics Symposium (HAPTICS), 2016 IEEE, 2016.

[49] W. Tang, N. Chen, and J. Z. et al., "Characterization of tactile perception and optimal exploration movement," Tribology Letters, vol. 58, no. 28, 2015.

[50] R. Fagiani and M. Barbieri, "Modeling of finger-surface contact dynamics," Tribology International, no. 74, 2014.
[51] R. Fagiani, F. Massi, E. Chalet, Y. Berthier, and A. Akay, "Tactile perception by friction induced vibrations," Tribology International, vol. 44, pp. 1100-1110, 2011.

[52] S. Bensmaia and M. Hollins, "The vibrations of texture," Somatosensory and Motor Research, vol. 11, 2011.

[53] I. R. Summers, P. G. Cooper, P. Wright, D. A. Gratton, P. M. Milnes, and B. H. Brown, "Information from time-varying vibrotactile stimuli," Journal of Acoustical Society of America, vol. 102, no. 6, pp. 3686-3696, 1997.

[54] S. A. Cholewiak, K. Kim, H. Z. Tan, and B. D. Adelstein, "A frequency domain analysis of haptic gratings," IEEE Transactions on Haptics, vol. 3, no. 1, pp. 3-14, 2010.

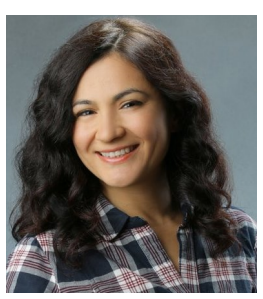

Yasemin Vardar Yasemin Vardar is currently a PhD candidate in mechanical engineering at Koç University, Istanbul. She received her BSc degree in mechatronics engineering from Sabanc University, Istanbul in 2010. Then, she received her MSc degree in systems and control from Eindhoven University of Technology in 2012 Before starting her PhD study, she conducted research on control of high precision systems in ASML, Philips, and TNO Eindhoven. Her research interests are haptic science and applications, mechatronics, and control.

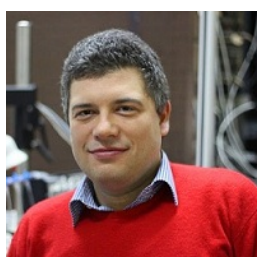

Burak Güçlü received the BS degree in contro and computer engineering from Istanbul Technical University, Turkey, in 1997. Then, he received his MS degree in bioengineering and the $\mathrm{PhD}$ degree in neuroscience, both from Syracuse University, New York, in 1999 and 2003 respectively, for his work on the physiology, psychophysics, and modeling of the sense of touch. During his postdoctoral research, he studied species-specific vocalizations and recorded from the prefrontal cortex of awake-behaving macaque monkeys at the medical school of University of Rochester. He has been a faculty member of the Institute of Biomedical Engineering at Bogazici University since 2004, where he is also the director of the Tactile Research Lab. His current research focuses on the transformation of tactile information from periphery to cortex in rats and somatosensory neuroprostheses.

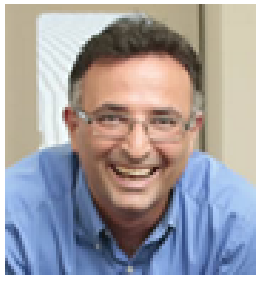

Cagatay Basdogan Cagatay Basdogan re ceived the PhD degree in mechanical engineering from Southern Methodist University in 1994 $\mathrm{He}$ is a faculty member in the mechanical engineering and computational sciences and engineering programs of Koc University, Istanbul, Turkey. He is also the director of the Robotics and Mechatronics Laboratory at Koc University. Before joining Koc University, he worked at NASA-JPL/Caltech, MIT, and Northwestern University Research Park. His research interests include haptic interfaces, robotics, mechatronics, biomechanics, medica simulation, computer graphics, and multi-modal virtual environments. $\mathrm{He}$ is currently the associate editor in chief of IEEE Transactions on Haptics and serves in the editorial boards of IEEE Transactions on Mechatronics, Presence: Teleoperators and Virtual Environments, and Computer Animation and Virtual Worlds journals. 